\title{
Advances in POEMS treatment and the need to define standardised outcome measures.
}

\section{Authors and affiliations}

S Keddie ${ }^{1,3}$, S D'Sa ${ }^{2}$, MP Lunn ${ }^{1,3}$

1. MRC Centre for Neuromuscular Disease, National Hospital of Neurology and Neurosurgery and Department of Molecular Neuroscience, UCL Institute of Neurology, Queen Square, London, UK.

2. Cancer Division, University College London Hospitals NHS Foundation Trust, London, UK

3. Neuroimmunology and CSF laboratory, Institute of Neurology, National Hospital for Neurology and Neurosurgery, University College London NHS Foundation Trust

\section{Corresponding author}

Dr Michael Lunn

MRC Centre for Neuromuscular Diseases,

National Hospital for Neurology and Neurosurgery,

Queen Square,

London

WC1N 3BG UK

Phone 1: +44 (0)2034483812

Fax 1: +44 (0)2034483797

Email: michaellunn@nhs.net

\section{Funding}

Dr Keddie is funded by an ABN/Guarantors of Brain Clinical Training Research Fellowship.

Dr Michael Lunn is supported by the National Institute for Health Research, University College London Hospitals, Biomedical Research Centre. 
No financial interests exist related to this publication.

\section{Conflicts of interest}

None

\section{Authorship}

Dr Keddie and Lunn designed the letter, Dr Keddie wrote the paper and Dr D'Sa and Dr Lunn provided several drafts/edits. All authors approved the final version. 
POEMS syndrome has been considered a rare 'cousin' of multiple myeloma with malignant but subtler monoclonal plasma cell proliferation and more aggressive cytokine driven end organ damage. New therapeutics for POEMS trickle down from large multicentre randomised controlled trials in multiple myeloma with the hope of similar efficacy, despite there being very clear but poorly understood differences in pathogenesis between the two. The face of POEMS treatment is however changing and POEMS is developing a life of its own; Li and colleagues have conducted the first clinical trial using melphalan for POEMS syndrome (Li et al., 2011), Misawa et al published their data from a randomised double-blind placebo-controlled trial of thalidomide treatment in 2016 (Misawa et al., 2016), and Nozza et al using Lenalidomide in a prospective open-label trial last year (Nozza et al., 2017). Clinical trials are underway studying lenalidomide in relapsed disease and the protease inhibitor Ixazomib.

All the studies above have shown significant results in a variety of different outcomes. Li demonstrated an $80 \%$ haematological response rate, with almost $40 \%$ of participants achieving complete response. Misawa studied Vascular Endothelial Growth Factor (VEGF) levels at 24 weeks compared to baseline, demonstrating significantly reduced VEGF levels in thalidomide treated patients compared to placebo. Nozza et al designed a 'Clinical Response Evaluation Scale' (CRES). Their CRES grades 10 POEMS features and attributes a score of 0-2 to each (total 0-20) dependent on severity. They illustrated improvements in CRES score and neuropathy features (using the Overall Neuropathy Limitations Score, ONLS) in their cohort of 18 patients treated with Lenalidomide. All the results above sound very promising, but also raise important questions for clinicians and patients selecting the most effective therapy.

The first question concerns outcome scores. With different studies using different methods to define treatment response, how does one compare results? How does a drop in VEGF compare to a complete haematological response? How does ONLS improvement of 1 point compare to CRES reduction of 3 ? Such seemingly useful scores become confusing and meaningless without an appropriate linear unified scale, and any possibility of statistically valid treatment comparisons in meta-analyses become futile. Secondly, what assessment criterion most accurately measures clinical outcome? Wang et al have recently demonstrated that plasma cells show remarkable VEGF expression both in mRNA and protein levels supporting the concept that plasma cells are responsible for downstream pathogenic effects (Wang et al., 2016). In multiple myeloma, validated haematological criteria have been defined, which allow for the identification of patients at high risk of progression (Rajkumar et al., 2014). Such criteria have been adapted for POEMS syndrome, but how important is haematological response in POEMS? POEMS manifests as a subtler monoclonal disorder, in which immune mediated cytokine proliferation appears to cause end-organ damage, rather than the proposed pathological mechanisms in myeloma. Additionally, the M-protein in POEMS is often low level and measurable at the lower limit of assay detection, if at all, making myeloma response criteria often inapplicable.

VEGF appears to function as an accurate and sensitive biomarker for disease identification and response to treatment in POEMS. It does not however correlate with disease severity, and mild phenotypes can have levels in the several thousands of $\mathrm{pg} / \mathrm{ml}$, and severely unwell patients just over normal. Although VEGF treatment response is usually dramatic, it may be disappointing, and does 
not linearly predict degree of improvement. This can be evenmore confusing in the Castleman variant of POEMS, in which neuropathy is often very mild. We have $9 / 80$ Castleman variant patients in our cohort, of which the median ONLS is $3 / 12$ conveying a mild loss of function, whereas median presenting VEGF level was 7669 (range 1102-11,000) demonstrating this discordance. Absolute VEGF levels do not always correlate well with disease activity,(Wang et al., 2017) however if combined with haematological response may be more accurate.(Zhao et al., 2018).

Neuropathy in POEMS syndrome results in severe morbidity and disability. Multiple neuropathy severity scales exist, (Allen et al., 2017) but such scoring systems were designed for patients with a range of inflammatory neuropathies, most prominently Chronic Inflammatory Demyelinating Polyneuropathy (CIDP). POEMS neuropathy fits in the inflammatory group, but the neuropathy is not the same. For example, pain is a prominent feature in POEMS syndrome often requiring neuropathic analgesia. CIDP tends to affect proximal muscles as an early feature, unlike POEMS which is lengthdependant. Scoring systems not designed for very specific diseases may not accurately capture severity or change in POEMS neuropathy. Secondly, POEMS neuropathy takes a long time to change and therefore cannot be very useful as an accurate predictor of response in the months immediately following treatment.

Degree of organ involvement appears to influence outcome in POEMS. Age over 50, pulmonary hypertension, pleural effusion and glomerular filtration rate were determined as factors which could estimate the 5 and 10 year overall survival, identified via a multivariate Cox hazards model of 362 POEMS cases (Wang et al., 2017). Kourelis et al identified age, albumin level and complete haematological remission as the only factors associated with progression (Kourelis et al., 2016). Inconsistencies in different cohorts make organ response criteria difficult to define. More work needs to be undertaken likely with multi-international centre cohorts to identify reliable prognosticating factors, and whether improvements in such lead to improved outcomes.

POEMS is a disease with different but equally confounding intricacies compared to myeloma. The pathogenesis, treatment response and long-term remission of both entities appear linked to the presence of a plasma cell disorder, but both have a range of multi-system downstream effects which clearly influence outcome. Looking forward, to design clinical trials in POEMS in which therapies can be assessed and compared in systematic meta-analysis, more robust, accurate and internationally recognised response criteria which accurately reflect disease severity and change are urgently needed. To do so, collaborative networks are required, to combine patient numbers for statistically powered analysis. Although such efforts appear complex, practically limiting and likely to take many years, one thing POEMS clinicians can learn from the world of myeloma is that it is an achievable and worthwhile endeavour. 


\section{References}

Allen, J. A., Gelinas, D. F., Lewis, R. A., Nowak, R. J. and Wolfe, G. I. (2017) 'Optimizing the Use of Outcome Measures in Chronic Inflammatory Demyelinating Polyneuropathy', US Neurology, 13(1), p. 26. doi: 10.17925/USN.2017.13.01.26.

Kourelis, T. V., Buadi, F. K., Gertz, M. A., Lacy, M. Q., Kumar, S. K., Kapoor, P., Go, R. S., Lust, J. A., Hayman, S. R., Rajkumar, V., Zeldenrust, S. R., Russell, S. J., Dingli, D., Lin, Y., Leung, N., Hwa, Y. L., Gonsalves, W., Kyle, R. A. and Dispenzieri, A. (2016) 'Risk factors for and outcomes of patients with POEMS syndrome who experience progression after first-line treatment', Leukemia, 30(5), pp. 10791085. doi: 10.1038/leu.2015.344.

Li, J., Zhang, W., Jiao, L. and Duan, M. (2011) 'Combination of melphalan and dexamethasone for patients with newly diagnosed POEMS syndrome', Blood, 117(24), pp. 6445-6450. doi: 10.1182/blood-2010-12-328112.An.

Misawa, S., Sato, Y., Katayama, K., Nagashima, K., Aoyagi, R., Sekiguchi, Y., Sobue, G., Koike, H., Yabe, I., Sasaki, H., Watanabe, O., Takashima, H., Nishizawa, M., Kawachi, I., Kusunoki, S., Mitsui, Y., Kikuchi, S., Nakashima, I., Ikeda, S. ichi, Kohara, N., Kanda, T., Kira, J. ichi, Hanaoka, H. and Kuwabara, S. (2016) 'Safety and efficacy of thalidomide in patients with POEMS syndrome: a multicentre, randomised, double-blind, placebo-controlled trial', The Lancet Neurology. Elsevier Ltd, 15(11), pp. 1129-1137.

Nozza, A., Terenghi, F., Gallia, F., Adami, F., Briani, C., Merlini, G., Giordano, L., Santoro, A. and Nobile-Orazio, E. (2017) 'Lenalidomide and dexamethasone in patients with POEMS syndrome: Results of a prospective, open-label trial', British Journal of Haematology, (October), pp. 748-755.

Rajkumar, S. V., Dimopoulos, M. A., Palumbo, A., Blade, J., Merlini, G., Mateos, M. V., Kumar, S., Hillengass, J., Kastritis, E., Richardson, P., Landgren, O., Paiva, B., Dispenzieri, A., Weiss, B., LeLeu, X., Zweegman, S., Lonial, S., Rosinol, L., Zamagni, E., Jagannath, S., Sezer, O., Kristinsson, S. Y., Caers, J., Usmani, S. Z., Lahuerta, J. J., Johnsen, H. E., Beksac, M., Cavo, M., Goldschmidt, H., Terpos, E., Kyle, R. A., Anderson, K. C., Durie, B. G. M. and Miguel, J. F. S. (2014) 'International Myeloma Working Group updated criteria for the diagnosis of multiple myeloma', The Lancet Oncology. Elsevier Ltd, 15(12), pp. e538-e548.

Wang, C., Huang, X.-F., Cai, Q.-Q., Cao, X.-X., Cai, H., Zhou, D. and Li, J. (2016) 'Remarkable expression of vascular endothelial growth factor in bone marrow plasma cells of patients with POEMS syndrome.', Leukemia research. Elsevier Ltd, 50, pp. 78-84.

Wang, C., Huang, X.-F., Cai, Q.-Q., Cao, X.-X., Duan, M.-H., Cai, H., Zhou, D.-B. and Li, J. (2017) 'Prognostic study for overall survival in patients with newly diagnosed POEMS syndrome', Leukemia, 31(1), pp. 100-106.

Zhao, H., Cai, H., Wang, C., Huang, X., Cao, X., Zhang, L., Zhou, D. and Li, J. (2018) 'Prognostic value of serum vascular endothelial growth factor and hematological responses in patients with newlydiagnosed POEMS syndrome', Blood Cancer Journal. Springer US, 8(4), p. 37. 